\title{
A survey on transarterial chemoembolization refractoriness and a real-world treatment pattern for hepatocellular carcinoma in Korea
}

\author{
Jae Seung Lee ${ }^{1}$, Beom Kyung Kim ${ }^{1,2,3}$, Seung Up Kim ${ }^{1,2,3}$, Jun Yong Park ${ }^{1,2,3}$, Sang Hoon Ahn ${ }^{1,2,3}$, Jin Sil Seong ${ }^{3,4}$, \\ Kwang-Hyub Han ${ }^{1,2,3}$, and Do Young Kim ${ }^{1,2,3}$ \\ 'Department of Internal Medicine and ${ }^{2}$ Institute of Gastroenterology, Yonsei University College of Medicine, Seoul; ${ }^{3}$ Yonsei Liver Center, \\ Severance Hospital, Seoul; ${ }^{4}$ Department of Radiation Oncology, Yonsei University College of Medicine, Seoul, Korea
}

Background/Aims: Transarterial chemoembolization (TACE) is a standard treatment for intermediate-stage hepatocellular carcinoma (HCC), but there is much controversy about TACE refractoriness. The aim of this study was to identify trends in the actual clinical application of TACE and recognition of TACE refractoriness by Korean experts.

Methods: In total, 17 questionnaires on TACE refractoriness were administered to 161 clinicians via an online survey. Multiple answers were allowed for some questions.

Results: Most clinicians agreed that there is a need for standardization of TACE application through specific scoring systems $(n=124,77.0 \%)$. TACE refractoriness was predominantly expected by participants when recurrences were detected within 1 month $(n=70,43.5 \%)$, there were 4 to 6 tumors $(n=77,47.8 \%)$, the maximal tumor size was $3-5 \mathrm{~cm}$ $(n=49,30.4 \%)$, and when there was insufficient tumor necrosis despite TACE being repeated more than three times $(n=78$, $48.4 \%)$. Overall, sorafenib therapy $(n=137)$ and radiotherapy $(n=114)$ were preferred when repeated TACE was considered ineffective.

Conclusions: Treatment of HCC is often based on the clinical judgment of clinicians because of the heterogeneity among individuals. Experts need to continue discussions on the standardization and sub-classification of HCC treatment guidelines in Korea. (Clin Mol Hepatol 2020;26:24-32)

Keywords: Liver neoplasms; Carcinoma, Hepatocellular; Embolization, Therapeutic; Surveys and questionnaires

\section{Study Highlights}

This survey focused to investigate the transarterial chemoembolization (TACE) treatment strategy of Korean clinicians for liver cancer. There were various opinions and differences of domestic medical staff about TACE refractoriness according to hospital size and location, but most of them showed convergence pattern. These results would help to establish the definition of TACE refractoriness and assist in the liver cancer treatment strategies in Korea.

\section{Abbreviations:}

ART score, the assessment for retreatment with transarterial chemoembolization score; BCLC, Barcelona-clinic liver cancer; HAP score, the Hepatoma Arterialembolization Prognostic score; HCC, hepatocellular carcinoma; TACE, transarterial chemoembolization

\section{Corresponding author : Do Young Kim}

Department of Internal Medicine, Yonsei University College of Medicine, 50-1 Yonsei-ro, Seodaemun-gu, Seoul 03722, Korea

Tel: +82-2-2228-1992, Fax: +82-2-383-6884

E-mail: DYK1025@yuhs.ac

https://orcid.org/0000-0002-8327-3439 


\section{INTRODUCTION}

Transarterial chemoembolization (TACE) is being widely used as a non-surgical therapy for the simultaneous treatment of chemotherapy and selective ischemia for hepatocellular carcinoma (HCC). On the basis of a higher level of evidence, TACE has been proven to improve the survival rate for patients with intermediate-stage HCC, defined as Barcelona-clinic liver cancer (BCLC) stage B HCC, for which patients are not candidates for curative strategies such as surgical resection, liver transplantation, and local ablative therapies. $^{1-4}$

Depending on the nature of the tumor, including size, number, growth pattern, and anatomical location, the treatment response is not always easy to obtain from a single session, showing differences in outcomes between reports. ${ }^{5,6}$ Thus, repeated TACEs are often performed to achieve a sufficient outcome. However, the method for predicting "TACE refractoriness" that does not show a therapeutic response even after repeated TACE treatments is still unclear. Enhanced criteria of TACE refractoriness were proposed by Japanese experts, which considered insufficient treatment response, increase in tumor number, new structural invasion or metastasis, and change in tumor markers. ${ }^{7}$ In the recent Korean guideline, ${ }^{8-10}$ sorafenib treatment was recommended if the stage progressed despite more than three repeated TACE treatments within 6 months.

However, in practice, there are too many heterogeneous situations to select a consistent treatment strategy; therefore, the clinical decision of the primary physician often plays a larger role than the formal guidelines in determining the appropriate time to try or switch to other therapies including radiotherapy, radiofrequency ablation, and sorafenib.

Therefore, we conducted an online survey and summarized the results below to identify the trends in the actual clinical application of TACE and the recognition of TACE refractoriness among the clinicians treating HCC in Korea.

\section{MATERIALS AND METHODS}

From November 9, 2017 to December 16, 2017, a total of 161 liver cancer clinicians in Korea were enrolled, including 121 gastroenterologists, 15 surgeons, five radiation oncologists, one hematologist, and 19 medical doctors of other fields (Table 1). All participants were working in tertiary medical centers where treatment procedures were performed via an intra-arterial route. Each indi- vidual accessed the online survey and selected his/her desired answers among the 17 multiple-choice questions. For 11 of the 17 questions, the participants were allowed to select multiple answers. All participants had worked in their fields for more than 10 years.

To compare practice patterns according to the size of the hospital, participants were divided into two groups: the five highestvolume centers and the lower volume centers. Clinical practice patterns according to the location of the hospitals were also compared by dividing participants into two groups: who were located in metropolitan areas (Seoul and Gyeonggi provinces) that had chance to easily access to the five high-volume centers, and who were located in other provinces.

The General Rules for the Clinical and Pathological Study of Primary liver cancer, developed by the Liver Cancer Study Group of Japan, were used to classify portal vein tumor thrombosis. ${ }^{10}$ According to these rules, the definition of $\mathrm{Vp} 3$ was the presence of a tumor thrombus in the first branches of the portal vein, and the definition of Vp4 was the presence a tumor thrombus in the main trunk of the portal vein and/or the contra-lateral portal vein branch to the primarily involved lobe.

Statistical analyses were conducted using the IBM SPSS Statistics software, version 23.0 (IBM Corp., Armonk, NY, USA), and a two-sided $P$-value of $<0.05$ was considered significant. The Chisquare test and Fisher's exact test, as appropriate, were applied to compare qualitative variables.

\section{RESULTS}

Baseline information of the participants are summarized in Table 1.

Table 1. Baseline information of the participants $(n=161)$

\begin{tabular}{lc}
\hline Variable & Value \\
\hline Male sex & $128(79.5)$ \\
\hline Clinicians working in the high-volume centers & $42(26.1)$ \\
Clinicians working in Seoul and Gyeonggi province & $120(74.5)$ \\
Specialty & \\
Gastroenterology and hepatology & $121(75.2)$ \\
Surgery & $15(9.3)$ \\
Radiation oncology & $5(3.1)$ \\
Hemato-oncology & $1(0.6)$ \\
Others & $19(11.8)$ \\
\hline
\end{tabular}

Variables are presented as $n(\%)$. 
Of $128(79.5 \%)$ male and 33 (20.5\%) female participants, 42 $(26.1 \%)$ clinicians were working in the five high-volume centers, and $120(74.5 \%)$ clinicians were working in the medical centers located in Seoul and Gyeonggi provinces.

\section{Questions about the overall perception of TACE}

The survey asked participants the following three yes/no questions.

A Standardization of TACE application in Korea is necessary through specific scoring systems such as the HAP score and ART score.

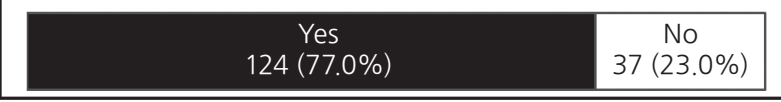

B The effect of TACE would be different depending on individual and tumor characteristics.

\section{Yes, 157 \\ $(97.5 \%)$ \\ Not certain $4(2.5 \%)$}

Sub-classification of the intermediate stage is necessary where TACE is recommended as a standard therapy.

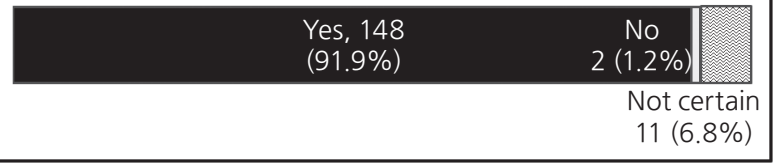

Figure 1. Voting results for three questions for $(A)$ the standardization of transarterial chemoembolization (TACE) application, (B) the possibility of different TACE effects, and (C) the need for sub-classification for effective TACE. HAP score, the Hepatoma Arterial-embolization Prognostic score; ART score, the Assessment for Retreatment with Transarterial chemoembolization score.
Q1. Standardization of TACE application in Korea is necessary through specific scoring systems such as the Hepatoma Arterialembolization Prognostic score (HAP score) and assessment for retreatment with TACE score (ART score).

Q2. The effect of TACE would be different depending on individual and tumor characteristics.

Q3. Sub-classification of the intermediate stage is necessary where TACE is recommended as a standard therapy.

Most of the clinicians agreed that there are needs for the standardization of TACE application through specific scoring systems ( $n=124,77.0 \%)$ and the sub-classification of the intermediate stage HCC where TACE is recommended as standard therapy ( $n=148,91.9 \%$ ). Moreover, most of the respondents ( $n=157$, 97.5\%) expected that the effect of TACE would be different depending on individual and tumor characteristics. There was no difference in the answers to the questions depending on the size and location of the hospital, except that the clinicians working far outside of Seoul more strongly raised the need for scoring systems (Fig. 1, Table 2).

\section{Factors affecting TACE treatment response}

The survey asked participants the following three questions that allowed multiple answers (Fig. 2, Table 3).

Q4. The patient characteristics affecting the response to TACE. Many clinicians responded that many variables would affect TACE response, especially the tumor size ( $n=145,90.1 \%)$, number of tumors ( $n=111,68.9 \%)$, and tumor shape, such as a nodular or infiltrating type ( $n=116,72.0 \%$ ) (Fig. 2A). Clinicians working in the high-volume centers showed a significant tendency to respond

Table 2. Responses to three questions $(n=161)$

\begin{tabular}{|c|c|c|c|c|c|c|c|c|}
\hline \multirow{2}{*}{ Question } & \multirow{2}{*}{ Answer } & \multirow{2}{*}{ No. } & \multicolumn{3}{|c|}{ High-volume centers ( $n=42)$} & \multicolumn{3}{|c|}{ Centers in metropolitan $(n=120)$} \\
\hline & & & Yes & No & $P$-value & Yes & No & $P$-value \\
\hline \multirow[t]{2}{*}{ Q1 } & Yes & $124(77.0)$ & $32(76.2)$ & $92(77.3)$ & 0.882 & $86(71.7)$ & 38 (92.7) & 0.005 \\
\hline & No & $37(23.0)$ & 10 (23.8) & 27 (22.7) & & $34(28.3)$ & $3(7.3)$ & \\
\hline \multirow[t]{2}{*}{ Q2 } & Yes & $157(97.5)$ & $40(95.2)$ & $117(98.3)$ & 0.279 & $116(96.7)$ & $41(100)$ & 0.573 \\
\hline & Not certain & $4(2.5)$ & $2(4.8)$ & $2(1.7)$ & & $4(3.3)$ & $0(0.0)$ & \\
\hline \multirow[t]{3}{*}{ Q3 } & Yes & 148 (91.9) & 39 (92.9) & 109 (91.6) & 0.962 & $108(90.0)$ & 40 (97.6) & 0.123 \\
\hline & No & $2(1.2)$ & $1(2.4)$ & $1(0.8)$ & & $2(1.7)$ & $0(0.0)$ & \\
\hline & Not certain & $11(6.8)$ & $2(4.8)$ & $9(7.6)$ & & $10(8.3)$ & $1(2.4)$ & \\
\hline
\end{tabular}

Values are presented as $n(\%)$ unless otherwise indicated. 'Q1' is 'standardization of transarterial chemoembolization (TACE) application in Korea is necessary through the specific scoring systems such as the Hepatoma Arterial-embolization Prognostic score (HAP score) and, the Assessment for Retreatment with TACE score (ART score)'. 'Q2' is 'the effect of TACE would be different depending on individual and tumor characteristics'. 'Q3' is 'sub-classification of the intermediate stage is necessary where TACE is recommended as a standard therapy'. $P$-value was calculated using chi-square test and Fisher's exact test. 
A Which of the following patient characteristics do you think the response to TACE is affected by? (multiple answers is allowed)

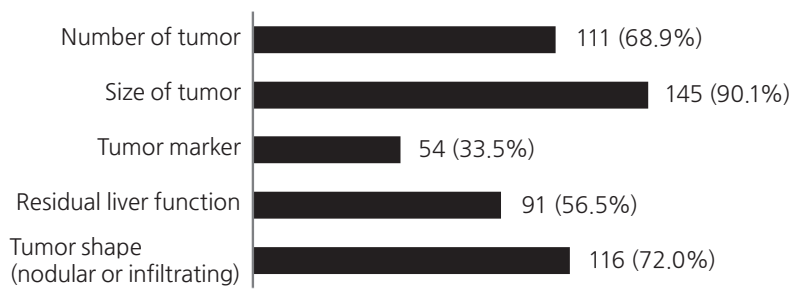

Based on your experience, which treatment have you chosen for the cases thought to be poor responders to TACE treatment? (multiple answers is allowed)

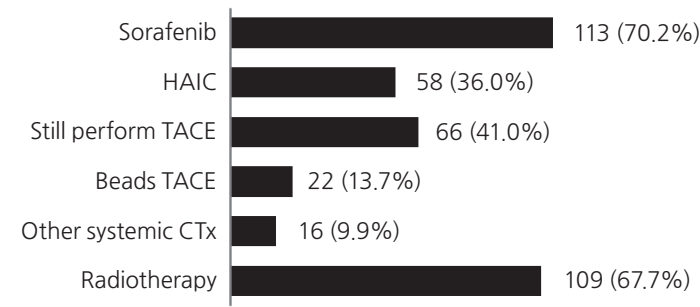

C What features do you think that make repeat TACE ineffective when performed in patients with localized masses? (multiple answers is allowed)

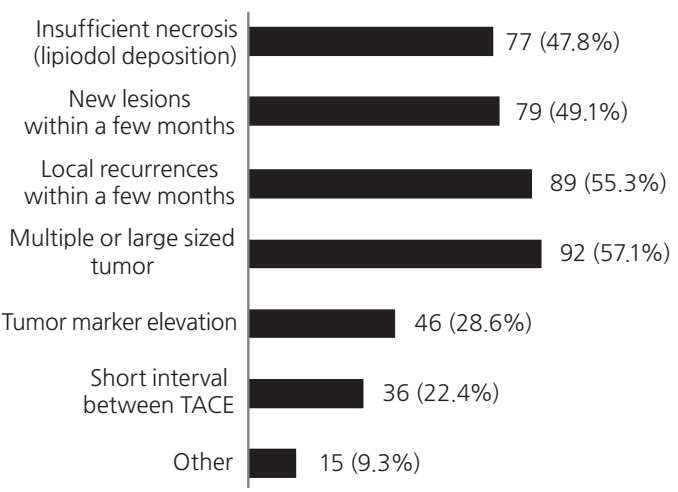

Figure 2. Clinical responses to three questions allowing multiple answers for $(A)$ individual characteristics that affect the response to transarterial chemoembolization (TACE) treatment, (B) subsequent treatments after insufficient TACE, $(C)$ and the possible features that make TACE treatment ineffective. HAIC, hepatic arterial infusion chemotherapy; CTx, chemotherapy.

that the tumor marker (50.0\% vs. $27.7 \%, P=0.013$ ) and tumor shape $(88.1 \%$ vs. $66.4 \%, P=0.009)$ were important (Table 3).

Q5. Preferred treatment, based on each clinician's experience, for the patients thought to be poor responders to TACE treatment. Sorafenib therapy $(n=113,70.2 \%)$ and radiotherapy $(n=109$, $67.7 \%$ ) were predominantly considered when TACE was not ex- pected to be effective and there were also many responses to consider TACE anyway ( $n=66,41.0 \%)$ or hepatic arterial infusion chemotherapy (HAIC) $(n=58,36.0 \%)$ (Fig. 2B). Sorafenib was preferred by clinicians working far outside of Seoul (65.8\% vs. 82.9, $P=0.039$ ) (Table 3).

Q6. The features that cause repeated TACE to be ineffective when used in patients with tumors localized in the liver. When performing TACE in a patient with a localized HCC in the liver, they suggested that TACE was unlikely to be effective in cases with a higher number of larger sized of tumors ( $n=92,57.1 \%)$, recurrent tumors, new lesions within a few months after the previous TACE treatment ( $n=89,55.3 \%$ and $n=79,49.1 \%$, respectively), and insufficient lipiodol uptake after TACE ( $n=77,47.8 \%$ ) (Fig. 2C). The response to tumor marker elevation ( $n=46,28.6 \%$ ) was significantly higher in high-volume centers ( $40.5 \%$ vs $24.4 \%$ ) (Table 3 ).

\section{Expectations for TACE refractoriness}

There were four questions regarding the situations where TACE refractoriness was expected (Fig. 3, Supplementary Table 1).

Q7. How long do you think it would take to detect new lesions or recurrences after TACE in TACE refractoriness?

Q8. If new lesions appear after TACE, how many tumors do you think are maladaptive for repeated TACE?

Q9. If local recurrences occur after TACE, how large is the maximal size of a tumor considered for TACE refractoriness?

Q10. How many times should there be insufficient necrosis or recurrences after repeated TACE for consideration as TACE refractoriness?

Participants replied as follows; TACE refractoriness was predominantly expected when new lesions or recurrence were detected at 1 month ( $n=70,43.5 \%), 3$ months ( $n=43,26.7 \%)$, and 2 months ( $n=29,18.0 \%)$ after the procedure, respectively. Clinicians working in high-volume centers $(66.7 \%$ vs. $35.3 \%$ for 1 month, $P=0.002)$ or centers near Seoul $(50.0 \%$ vs. $24.4 \%$ for 1 month, $P=0.045)$ preferred to determine TACE refractoriness at an earlier time point (Fig. 3A, Supplementary Table 1). Regarding the number of lesions, 4 to 6 lesions ( $n=77,47.8 \%)$, below 3 lesions ( $n=28,17.4 \%)$, and 7 to 10 lesions $(n=21,13.0 \%)$ were frequently selected (Fig. 3B). Regarding the size of the largest lesion, $3-5 \mathrm{~cm}(\mathrm{n}=49,30.4 \%)$ and $5-7 \mathrm{~cm}(\mathrm{n}=40,24.8 \%)$ were mostly chosen (Fig. 3C). Regarding the number of insufficient TACE or repeated new lesions, 3 times $(n=78,48.4 \%)$ and 2 times $(n=62$, $38.5 \%$ ) were mostly selected (Fig. 3D). 
Table 3. Responses to three questions allowing multiple answers

\begin{tabular}{|c|c|c|c|c|c|c|c|c|}
\hline \multirow{2}{*}{ Question } & \multirow{2}{*}{ Answer } & \multirow{2}{*}{ No. } & \multicolumn{3}{|c|}{ High-volume centers ( $n=42$ ) } & \multicolumn{3}{|c|}{ Centers in metropolitan $(n=120)$} \\
\hline & & & Yes & No & $P$-value & Yes & No & $P$-value \\
\hline \multirow[t]{5}{*}{ Q4 } & Number of tumor & $111(68.9)$ & $29(69.0)$ & $82(68.9)$ & 0.987 & $81(67.5)$ & $30(73.2)$ & 0.498 \\
\hline & Size of tumor & $145(90.1)$ & $39(92.9)$ & $106(89.1)$ & 0.565 & $106(88.3)$ & 39 (95.1) & 0.363 \\
\hline & Tumor marker & $54(33.5)$ & $21(50.0)$ & $33(27.7)$ & 0.013 & $44(36.7)$ & $10(24.4)$ & 0.151 \\
\hline & Residual liver function & $91(56.5)$ & $24(57.1)$ & $67(56.3)$ & 0.925 & $71(59.2)$ & $20(48.8)$ & 0.247 \\
\hline & Tumor shape (nodular or infiltrating) & $116(72.0)$ & 37 (88.1) & 79 (66.4) & 0.009 & $91(75.8)$ & $25(61.0)$ & 0.067 \\
\hline \multirow[t]{6}{*}{ Q5 } & Sorafenib & $113(70.2)$ & $32(76.2)$ & $81(68.1)$ & 0.322 & 79 (65.8) & $34(82.9)$ & 0.039 \\
\hline & HAIC & $58(36.0)$ & $13(31.0)$ & $45(37.8)$ & 0.426 & $44(36.7)$ & $14(34.1)$ & 0.772 \\
\hline & Still perform TACE & $66(41.0)$ & $13(31.0)$ & $53(44.5)$ & 0.124 & $52(43.3)$ & $14(34.1)$ & 0.302 \\
\hline & Beads TACE & $22(13.7)$ & $7(16.7)$ & $15(12.6)$ & 0.602 & $18(15.0)$ & $4(9.8)$ & 0.599 \\
\hline & Other systemic chemotherapy & $16(9.9)$ & $3(7.1)$ & $13(10.9)$ & 0.565 & $11(9.2)$ & $5(12.2)$ & 0.556 \\
\hline & Radiotherapy & $109(67.7)$ & $33(78.6)$ & $76(63.9)$ & 0.087 & $83(69.2)$ & $26(63.4)$ & 0.497 \\
\hline \multirow[t]{7}{*}{ Q6 } & Insufficient necrotic area & $77(47.8)$ & $16(38.1)$ & $61(51.3)$ & 0.142 & $61(50.8)$ & $16(39.0)$ & 0.191 \\
\hline & New lesions within a few months & 79 (49.1) & $23(54.8)$ & $56(47.1)$ & 0.391 & $53(44.2)$ & $26(63.4)$ & 0.033 \\
\hline & Local recurrences within a few months & $89(55.3)$ & $22(52.4)$ & $67(56.3)$ & 0.660 & $62(51.7)$ & $27(65.9)$ & 0.115 \\
\hline & Tumor size or number & $92(57.1)$ & $24(57.1)$ & $68(57.1)$ & 1.000 & $68(56.7)$ & $24(58.8)$ & 0.835 \\
\hline & Tumor marker elevation & $46(28.6)$ & $17(40.5)$ & $29(24.4)$ & 0.047 & $36(30.0)$ & $10(24.4)$ & 0.492 \\
\hline & Short interval between repeated TACE & $36(22.4)$ & $10(23.8)$ & 26 (21.8) & 0.793 & $23(19.2)$ & $13(31.7)$ & 0.096 \\
\hline & Others & $15(9.3)$ & $6(14.3)$ & $9(7.6)$ & 0.221 & $11(9.2)$ & $4(9.8)$ & 1.000 \\
\hline
\end{tabular}

Values are presented as $\mathrm{n}(\%)$ unless otherwise indicated. 'Q4' is 'the patient characteristics affecting the response to transarterial chemoembolization (TACE)'. 'Q5' is 'preferred treatment based on each clinicians' experience for the cases thought to be poor responders to TACE'. 'Q6' is 'the features that make repeated TACE ineffective when performed with tumors localized in the liver.' $P$-value was calculated using chi-square test and Fisher's exact test. HAIC, hepatic arterial infusion chemotherapy.

\section{Preferred treatment strategies after TACE in specific situations}

Participants replied to the questions that allowed multiple answers about the preferred treatment strategies after TACE in the following seven situations; Q11, suspicious TACE failure or refractoriness; Q12, multiple local recurrences with Child-Pugh class A; Q13, Vp3/4 portal vein thrombosis; Q14, main portal vein invasion; Q15, extrahepatic metastasis with Child-Pugh class A; Q16, extrahepatic metastasis with Child-Pugh class B; Q17, insufficient necrosis after at least two treatments of TACE with Child-Pugh class A (Fig. 4, Supplementary Table 2).

Overall, sorafenib ( $n=137,85.1 \%)$, radiotherapy $(n=114,70.8 \%)$ and HAIC $(n=62,38.5 \%)$ were preferred when repeated TACE was considered ineffective. Preferred treatment methods in the specific conditions after performing TACE were reported as follows: Repeat TACE ( $n=111,68.9 \%)$ and sorafenib ( $n=97,60.2 \%)$ for multiple intrahepatic recurrences with Child-Pugh class A. For
$\mathrm{Vp3} / 4$ thrombosis and main portal vein invasion, radiotherapy ( $n=127,78.9 \%$ and $n=138,85.7 \%$, respectively) and sorafenib ( $n=93,57.8 \%$ and $n=106,65.8 \%$, respectively) were preferred. Sorafenib ( $n=153,95.0 \%$ ) was preferred for extrahepatic recurrence with Child-Pugh class $A$. In the case of extrahepatic recurrence with Child-Pugh class B, hospice care $(n=74,46.0 \%)$ was preferred with other concurrent therapies (sorafenib $[n=65$, $40.4 \%]$, radiotherapy $[n=61,37.9 \%$ ] and other systemic chemotherapy $[n=51,31.7 \%]$, respectively). When the necrosis of tumors with Child-Pugh class A were insufficient after performing more than 2 treatments of TACE, radiotherapy $(n=113,70.2 \%)$, sorafenib $(n=94,58.4 \%)$ and repeat TACE $(n=75,46.6 \%)$ were preferred (Fig. 4). For local control of HCC with advanced stage and ChildPugh class A liver function, radiotherapy was preferred over repeated trials of TACE treatment in the high-volume centers (Q13, $P=0.008 ; \mathrm{Q} 14, P=0.009 ; \mathrm{Q} 17, P=0.011)$ or centers in the metropolitan areas (Q14, $P=0.032 ; \mathrm{Q17}, P=0.022$ ) (Supplementary Table 2). 


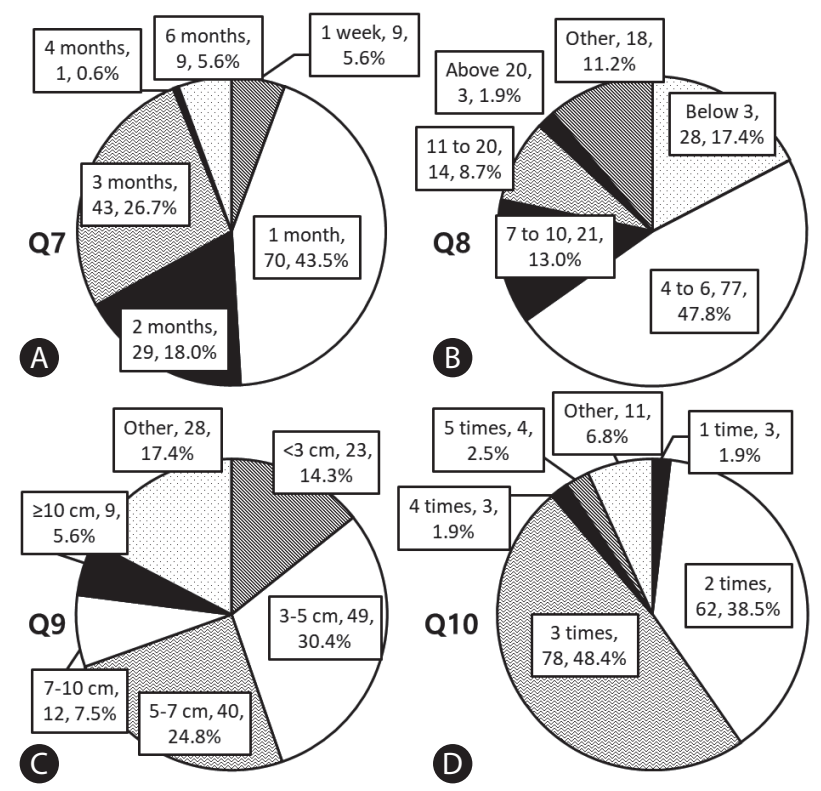

Figure 3. Answers to questions about transarterial chemoembolization (TACE) refractoriness. (A) Q7, how long do you think it would take to detect new lesions or recurrences after TACE in TACE refractoriness? (B) Q8, if new lesions appear after TACE, how many tumors do you think are maladaptive for repeated TACE? (C) Q9, if local recurrences occur after TACE, how large is the maximal size of tumors considered for TACE refractoriness? (D) Q10, how many times should there be insufficient necrosis or recurrences after repeated TACE for consideration as TACE refractoriness?

\section{DISCUSSION}

The effects of TACE on HCC and the changes in survival patterns may vary depending on the patient's residual liver function and the characteristics of the tumor itself, so it is often necessary to repeatedly perform TACE because a single trial does not obtain sufficient results in many cases. ${ }^{11,12}$ It is still controversial whether treatment should be changed during repeated TACE or whether the effect obtained at some time point will help predict a patient's survival. ${ }^{5,13}$ Although the recent $\mathrm{HCC}$ guideline by the Korean Liver Cancer Association suggested that progression of the disease after multiple trials of TACE treatment could be regarded as TACE refractoriness ${ }^{14}$ there has been no actual definition of TACE refractoriness that can help further establish a treatment plan.

Therefore, the survey was conducted to see how medical staff in Korea were actually applying TACE in their work fields. In this survey, tumor number, size, and shape (nodular or infiltrative type) were considered as factors to affect the response to TACE treatment. A short period (about 1-3 months) until the appearance of the new lesions or recurrences, the maximum size of 3-7 $\mathrm{cm}$ of local recurrence, and the insufficient necrosis or recurrences after 2-3 times of repeated TACE treatments were considered to be suitable for TACE refractoriness. For multiple local recurrence, participants responded that repeated TACE treatment could be performed. However, for patients with advanced stage or suspected TACE refractoriness, clinicians selected sorafenib and localized radiotherapy more frequently. Radiotherapy was more preferred to be performed by the clinicians in the large medical centers or centers with good accessibility to the larger centers, probably because the high-volume centers had sufficient experience and appropriate facilities to perform radiation therapy.

TACE is useful because it can minimize impairment of hepatic function while improving treatment response and survival rate. However, at a certain point in time, repeated TACE treatments could show refractory patterns that diminish its usefulness and result in necrosis of multifocal lesions, which could lead to aggravation of liver function and worse patient survival. ${ }^{7}$ Therefore, it is important to determine a precise definition of TACE refractoriness to maximize the effectiveness of TACE itself and other therapies.

Many efforts have been made to subclassify the intermediate stage or provide prediction models through other scoring systems. Sub-classification by applying the Milan criteria and up to 7 criteria had been proposed, while classifying HCC patients in the intermediate stage into B1, B2, B3, B4, and Quasi-C groups according to basal liver function and the presence of marginal portal thrombus. ${ }^{6}$ The HAP score began as a predictive model for the effectiveness of the first TACE by scoring serum albumin, serum total bilirubin, serum alpha-fetoprotein, and maximum tumor size. ${ }^{15}$ Moreover, the modified HAP-II scoring system, which additionally evaluated the presence of multiple tumors, showed efficacy in 280 patients with HCC in Korea. ${ }^{16}$ The ART score system, including the Child-Pugh score, serum aspartate aminotransperase, and response evaluation after the first TACE, ${ }^{17}$ has not shown efficacy in other studies of HCC patients with chronic hepatitis $\mathrm{C}$ in Japan, ${ }^{18}$ Italy, ${ }^{19}$ and France ${ }^{20}$ and chronic hepatitis B in Taiwan, ${ }^{21}$ and it was also ineffective in evaluating the efficacy of repeated drugeluting bead TACE for HCC patients in a Spanish multicenter study. ${ }^{22}$ However, combined application of the ART score after classification with the HAP score showed a significant efficacy in predicting TACE refractoriness in a recent multicenter study, and an algorithm for performing repeat TACE in early- and intermediate-stage HCC was suggested, and repeated TACE could be performed for patients with an ART score less than 2.5 after the first TACE. $^{23}$

Some recent studies have shown that obtaining a complete re- 
After TACE treatment, which treatment strategies are you considering in the following situations? (multiple answer is allowed)

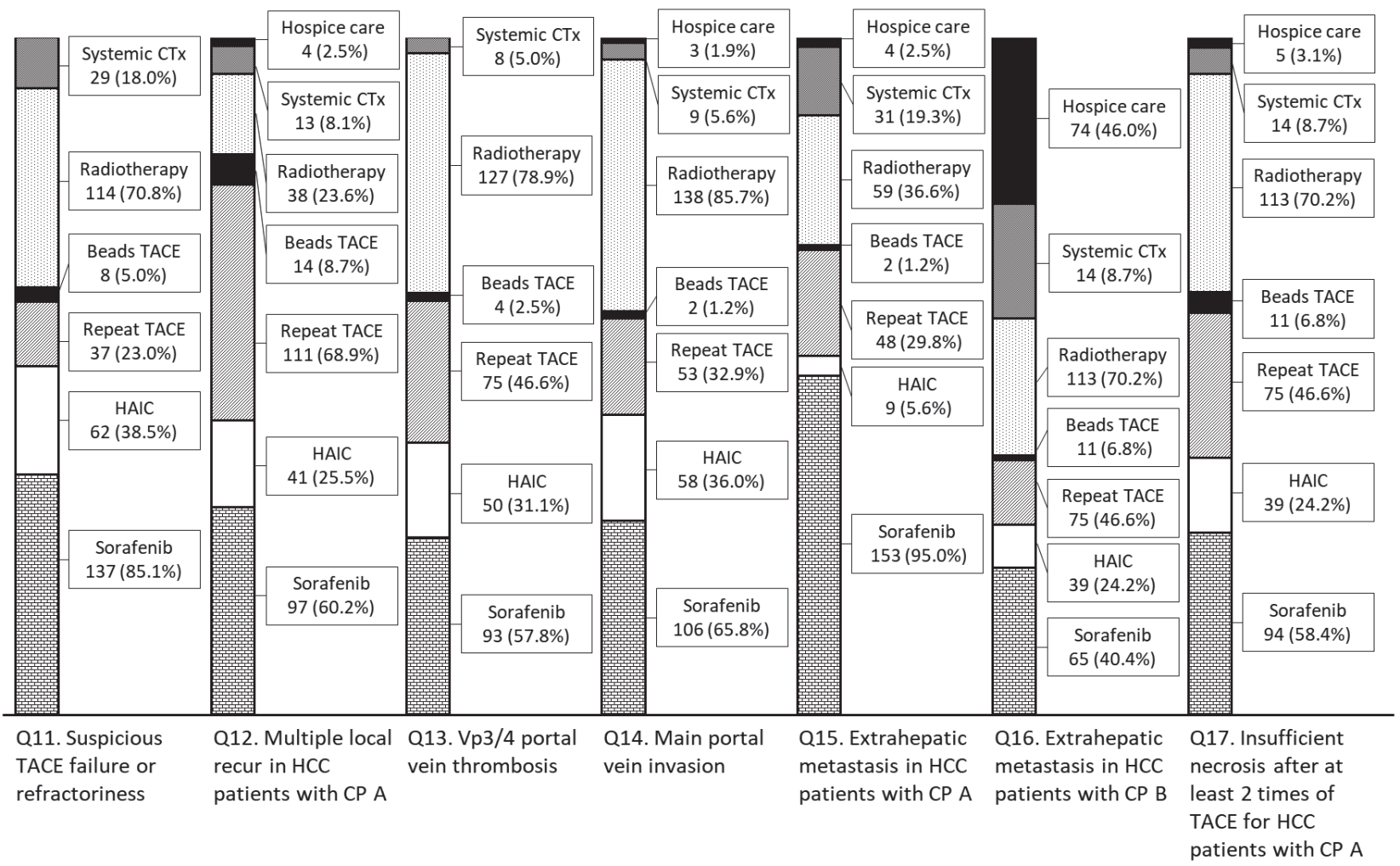

Figure 4. Voting results for questions about the treatment strategies after transarterial chemoembolization (TACE) for the participants considered in the following situations. CTx, chemotherapy; HAIC, hepatic arterial infusion chemotherapy; HCC, hepatocellular carcinoma; CP, Child-Pugh classification.

sponse at an earlier time strongly predicted a favorable survival outcome in patients with HCC. ${ }^{13,24,25}$ Moreover, BCLC stage, pretreatment alpha-fetoprotein level, and tumor number were also independent risk factors for overall survival along these studies. ${ }^{13,24}$ Regarding this, not only the on-treatment response but also baseline tumor characteristics and laboratory data could be used as official predictors for TACE refractoriness in the guideline. Assessing TACE refractoriness as quickly as possible could provide clinicians with the opportunity to make treatment strategies easier (such as localized radiotherapy, TACE, and concurrent therapies) and to increase the effectiveness of these next treatment strategies.

According to the results of this survey, the treatment of HCC favored by participants were not significantly different from the treatments recommended in the BCLC stage or proposed by the various scoring systems or sub-classifications as mentioned above. Of course, the management of HCC is difficult to simply standardize and the clinical judgment of the primary care physi- cian considering individual tumor characteristics, economic situation, and the familial environment is still important, but is difficult without sufficient clinical experience. Therefore, standardization of treatments that many physicians can use is still necessary. There is also a need for experts to continue to discuss the subdivision of guidelines for the treatment of HCC in Korea.

\section{Authors' contribution}

JS Lee and DY Kim: Data acquisition, analysis and interpretation, drafting of manuscript, and statistical analysis.

DY Kim: Study concept and design, data analysis and interpretation, drafting and critical revision of manuscript, and study supervision.

BK Kim, SU Kim, JY Park, SH Ahn, JS Seong, and KH Han: Critical revision of the manuscript.

All authors approved the final version of manuscript. 


\section{Conflicts of Interest}

Bayer supported the on-line survey (http://tacenow.com/survey).

\section{SUPPLEMENTARY MATERIALS}

Supplementary materials are available at Clinical and Molecular Hepatology website (http://www.e-cmh.org).

\section{REFERENCES}

1. Korean Liver Cancer Association, National Cancer Center. 2018 Korean Liver Cancer Association-National Cancer Center Korea Practice Guidelines for the management of hepatocellular carcinoma. Gut Liver 2019;13:227-299.

2. Burrel $M$, Reig M, Forner $A$, Barrufet $M$, de Lope $C R$, Tremosini $S$, et al. Survival of patients with hepatocellular carcinoma treated by transarterial chemoembolisation (TACE) using Drug Eluting Beads. Implications for clinical practice and trial design. J Hepatol 2012;56:1330-1335.

3. Bruix J, Sherman M; American Association for the Study of Liver Diseases. Management of hepatocellular carcinoma: an update. Hepatology 2011;53:1020-1022.

4. Kim BK, Kim SU, Park JY, Kim DY, Ahn SH, Park MS, et al. Applicability of $B C L C$ stage for prognostic stratification in comparison with other staging systems: single centre experience from long-term clinical outcomes of 1717 treatment-naive patients with hepatocellular carcinoma. Liver Int 2012;32:1120-1127.

5. Terzi E, Golfieri R, Piscaglia F, Galassi M, Dazzi A, Leoni S, et al. Response rate and clinical outcome of HCC after first and repeated CTACE performed "on demand". J Hepatol 2012;57:1258-1267.

6. Bolondi L, Burroughs A, Dufour JF, Galle PR, Mazzaferro V, Piscaglia $F$, et al. Heterogeneity of patients with intermediate (BCLC B) Hepatocellular Carcinoma: proposal for a subclassification to facilitate treatment decisions. Semin Liver Dis 2012;32:348-359.

7. Kudo M, Matsui O, Izumi N, Kadoya M, Okusaka T, Miyayama S, et al. Transarterial chemoembolization failure/refractoriness: JSHLCSGJ criteria 2014 update. Oncology 2014;87 Suppl 1:22-31.

8. McGlynn KA, Petrick JL, London WT. Global epidemiology of hepatocellular carcinoma: an emphasis on demographic and regional variability. Clin Liver Dis 2015;19:223-238.

9. Kim HY, Park JW, Joo J, Jung SJ, An S, Woo SM, et al. Severity and timing of progression predict refractoriness to transarterial chemoembolization in hepatocellular carcinoma. J Gastroenterol Hepatol 2012;27:1051-1056.

10. Kudo M, Izumi N, Ichida T, Ku Y, Kokudo N, Sakamoto M, et al.
Report of the 19th follow-up survey of primary liver cancer in Japan. Hepatol Res 2016;46:372-390.

11. Kim DY, Ryu HJ, Choi JY, Park JY, Lee DY, Kim BK, et al. Radiological response predicts survival following transarterial chemoembolisation in patients with unresectable hepatocellular carcinoma. Aliment Pharmacol Ther 2012;35:1343-1350.

12. Georgiades C, Geschwind JF, Harrison N, Hines-Peralta A, Liapi E, Hong $K$, et al. Lack of response after initial chemoembolization for hepatocellular carcinoma: does it predict failure of subsequent treatment? Radiology 2012;265:115-123.

13. Jung ES, Kim JH, Yoon EL, Lee HJ, Lee SJ, Suh SJ, et al. Comparison of the methods for tumor response assessment in patients with hepatocellular carcinoma undergoing transarterial chemoembolization. J Hepatol 2013;58:1181-1187.

14. Korean Liver Cancer Study Group (KLCSG); National Cancer Center, Korea (NCC). 2014 KLCSG-NCC Korea Practice Guideline for the management of hepatocellular carcinoma. Gut Liver 2015;9:267-317.

15. Kadalayil L, Benini R, Pallan L, O'Beirne J, Marelli L, Yu D, et al. A simple prognostic scoring system for patients receiving transarterial embolisation for hepatocellular cancer. Ann Oncol 2013;24:25652570.

16. Park Y, Kim SU, Kim BK, Park JY, Kim DY, Ahn SH, et al. Addition of tumor multiplicity improves the prognostic performance of the hepatoma arterial-embolization prognostic score. Liver Int 2016;36:100-107.

17. Sieghart W, Hucke F, Pinter M, Graziadei I, Vogel W, Müller C, et al. The ART of decision making: retreatment with transarterial chemoembolization in patients with hepatocellular carcinoma. Hepatology 2013;57:2261-2273.

18. Kudo M, Arizumi T, Ueshima K. Assessment for retreatment (ART) score for repeated transarterial chemoembolization in patients with hepatocellular carcinoma. Hepatology 2014;59:2424-2425.

19. Terzi E, Terenzi L, Venerandi L, Croci L, Renzulli M, Mosconi C, et al. The ART score is not effective to select patients for transarterial chemoembolization retreatment in an Italian series. Dig Dis 2014;32:711-716.

20. Adhoute X, Penaranda G, Naude S, Raoul JL, Perrier H, Bayle O, et al. Retreatment with TACE: the ABCR SCORE, an aid to the decisionmaking process. J Hepatol 2015;62:855-862.

21. Tseng CL, Lai WJ, Huang CJ, Huang YH, Su CW, Lee IC, et al. The effectiveness of ART score in selecting patients for transarterial chemoembolization retreatment: a cohort study in Taiwan. Medicine (Baltimore) 2015;94:e1659.

22. Pipa-Muñiz M, Castells L, Pascual S, Fernández-Castroagudín J, Díez-Miranda I, Irurzun J, et al. The ART-SCORE is not an effective tool for optimizing patient selection for DEB-TACE retreatment. A multicentre Spanish study. Gastroenterol Hepatol 2017;40:515-524.

23. Pinato DJ, Arizumi T, Jang JW, Allara E, Suppiah PI, Smirne C, et al. Combined sequential use of HAP and ART scores to predict survival 
outcome and treatment failure following chemoembolization in hepatocellular carcinoma: a multi-center comparative study. Oncotarget 2016;7:44705-44718.

24. Kim BK, Kim KA, Park JY, Ahn SH, Chon CY, Han KH, et al. Prospective comparison of prognostic values of modified Response Evaluation Criteria in Solid Tumours with European Association for the Study of the Liver criteria in hepatocellular carcinoma following chemoembolisation. Eur J Cancer 2013;49:826-834.

25. Gillmore R, Stuart S, Kirkwood A, Hameeduddin A, Woodward N, Burroughs AK, et al. EASL and mRECIST responses are independent prognostic factors for survival in hepatocellular cancer patients treated with transarterial embolization. J Hepatol 2011;55:13091316. 